\title{
La comunicación en el aula universitaria en el contexto del COVID-19 a partir de la videoconferencia con Google Meet
}

\section{(Communication at university classrooms in the context of COVID-19 by means of videoconferencing with Google Meet)}

\author{
Rosabel Roig-Vila \\ Mayra Urrea-Solano \\ Gladys Merma-Molina \\ Universidad de Alicante, UA (España)
}

\section{DOI: $\underline{\text { http://dx.doi.org/10.5944/ried.24.1.27519 }}$}

\section{Cómo referenciar este artículo:}

Roig-Vila, R., Urrea-Solano, M., y Merma-Molina, G. (2021). La comunicación en el aula universitaria en el contexto del COVID-19 a partir de la videoconferencia con Google Meet. RIED. Revista Iberoamericana de Educación a Distancia, 24(1), pp. 197-220. doi: http://dx.doi.org/10.5944/ried.24.1.27519

\section{Resumen}

La nueva realidad impuesta por el COVID-19 ha supuesto uno de los mayores desafíos que, hasta el momento, han tenido que hacer frente las universidades de tipo presencial, al tener que transitar de esta modalidad a una virtual y de emergencia. En concreto, la comunicación presencial en las aulas se ha tenido que apoyar en videoconferencias síncronas, las cuales han "ocupado" virtualmente un espacio que estaba regulado con anterioridad en las clases ordinarias. Todo ello ha requerido el uso de diversas aplicaciones tecnológicas, entre las cuales Google Meet ha sido una de las más utilizadas. En base a ello, este estudio se planteó con dos objetivos: (1) identificar las necesidades de comunicación que el alumnado universitario ha tenido durante el periodo de docencia virtual y (2) comprobar si Google Meet ha sido capaz de satisfacerlas. Para el logro de estos, se contó con la participación de 52 estudiantes del Grado de Maestro en Educación Primaria de la Universidad de Alicante. Se adoptó un enfoque metodológico mixto y para el tratamiento de los datos se emplearon el SPSS v. 25 y el AQUAD 7 , con los que se realizó un estudio descriptivo y un análisis de contenido respectivamente. De acuerdo con los resultados, si bien el profesorado ha utilizado una comunicación audiovisual síncrona, lo que se ajusta a las demandas del alumnado, el grado de satisfacción de este es notoriamente escaso. Algo similar sucede en el caso de Google Meet, ya que pese a reconocerle cierto grado de utilidad y disfrute, manifiestan haber tenido serias dificultades a la hora 
de utilizarlo. A la vista de tales hallazgos, se concluye señalando la necesidad de ajustar la respuesta tecnológica que desde la universidad se está dando a la situación provocada por el COVID-19.

Palabras clave: Google Meet; formación universitaria; estudiantes universitarios; COVID-19; educación virtual; comunicación.

\begin{abstract}
The new reality imposed by COVID-19 has meant one of the greatest challenges that face-to-face university teaching has had to confront, as these higher education centres were suddenly forced to change from a face-to-face format to a virtual, contingent kind of teaching. More precisely, face-to-face communication at classrooms now must rely on synchronous videoconferences which have digitally "occupied" the space that was previously regulated in ordinary lessons. It all required using a wide range of technological applications, amongst which Google Meet stood out as one of the most popular. Based on this, our study set itself two objectives: (1) identifying the communication needs that university students have felt throughout this period of virtual teaching; and (2) verifying the extent to which Google Meet succeeded in meeting those needs. Seeking to accomplish the aforesaid goals, we involved 52 students from the Degree in Primary Education Teacher Training imparted at the University of Alicante. A mixed methodological approach was adopted, the software programs utilized for data processing purposes being SPSS v. 25 and AQUAD 7, which enabled us to carry out a descriptive study and a content analysis, respectively. In view of the results, and although the teaching staff have used a synchronous type of audiovisual communication suited to the demands of students, the degree of satisfaction amongst the latter is remarkably low. Something similar happens when it comes to Google Meet because, despite recognizing that it has positive aspects such as a certain degree of usefulness and enjoyment, they claim to have had serious difficulties in using it. Such findings lead us to conclude that a compelling need exists to fine tune the technological response that universities are giving before the situation caused by COVID-19.
\end{abstract}

Keywords: Google Meet; university training; university students; COVID-19; virtual education; communication.

La pandemia iniciada en Wuhan el 29 de diciembre de 2019, conocida como COVID-19, ha puesto a las instituciones de Educación Superior de tipo presencial en una encrucijada sin parangón (UNESCO, 2020). Aunque con ligeras diferencias entre las medidas adoptadas por cada uno de los países, las elevadas cifras de fallecimientos y contagios han provocado, de manera obligada, que el aprendizaje abandone el aula física y pase a desarrollarse en el espacio virtual (Cecilio-Fernandes, Parisi, Santos y Sandars, 2020). Como consecuencia de ello, el profesorado se ha visto forzado a buscar nuevas estrategias y herramientas tecnológicas que permitan, en mayor o menor medida, adaptar la enseñanza y dar continuidad al curso lectivo 
(Barbosa y Amariles, 2019; Kerres, 2020). En este contexto, adquieren un especial protagonismo los recursos y aplicaciones que permiten una comunicación síncrona, por presentar una mayor semejanza con las clases presenciales (Carrick et al., 2017; Sandars et al., 2020; So, 2016); esto es, una mayor interacción entre el docente y el alumnado y entre los propios estudiantes, pese a que estos no compartan el mismo espacio (Cornelius, 2014). A ello, se ha de añadir, además, su potencialidad para reducir los sentimientos de aislamiento y soledad, que suelen ser habituales en la modalidad de aprendizaje virtual, así como la posibilidad de feedback inmediato que ofrecen (Chen, Dobinson y Kent, 2020; Trespalacios y Uribe-Florez, 2020).

Dentro de este marco, algunas de las herramientas que suelen utilizarse más frecuentemente son las videoconferencias online (Cendon, 2018; Chen, Liao, Chen y Lee, 2011; Cornelius, 2014; Infante-Moro, Infante-Moro y Gallardo-Pérez, 2019; Rao, 2019). Estas pueden ser definidas como sesiones o clases en las que el alumnado y el profesorado se conecta e interactúa entre sí en tiempo real, gracias al empleo de plataformas y aplicaciones virtuales (Gegenfurtner, Zitt y Ebner, 2020). Algunos de los softwares que se usan para llevarlas a cabo son Elluminate Live!, Wimba, Adobe Connect, Zoom, Blackboard Collaborate (Cornelius, 2014; Morrison, 2011; Villalón, Luna y García-Barrera, 2019) y, de reciente aparición, Google Meet, que viene a sustituir a Hangouts, servicio de mensajería instantánea y de video llamada de Google. El sencillo funcionamiento que presenta, así como las ventajas que ofrece, han provocado que Google Meet comience a ser utilizado cada vez más en el ámbito universitario. Un ejemplo de ello, es la reciente experiencia desarrollada por Budiana y Yutanto (2020) en Indonesia, quienes lo emplean para el aprendizaje de una segunda lengua con estudiantes universitarios. De acuerdo con los resultados de su estudio, la utilización de Google Meet no solo produjo una mejora considerable del nivel de rendimiento de estos, sino que también favoreció el desarrollo de la interacción y de la participación entre el grupo de alumnos.

En efecto, las potencialidades que presenta han motivado que Google Meet se convierta en una de las aplicaciones más recomendadas y utilizadas para hacer posible el tránsito de la presencialidad a la virtualidad, como consecuencia de la modalidad de enseñanza online impuesta por el COVID-19 (Taha, Abdalla, Wadi y Khalafalla, 2020). Con ella se espera, de alguna manera, venir a paliar algunas de las dificultades que los estudiantes reconocen estar experimentando para poder seguir su proceso de aprendizaje y, de ese modo, reducir las elevadas tasas de estrés y ansiedad que afirman están padeciendo (Franchi, 2020). En base a ello, y teniendo en cuenta la escasez de estudios que analizan el uso de la modalidad de enseñanza asíncrona en la Educación Superior (Cornelius, 2014; Morrison, 2011), este estudio se planteó con los objetivos de identificar las necesidades de comunicación que está experimentando el alumnado universitario en su proceso de aprendizaje virtual, como consecuencia del COVID-19, y valorar si el uso de Google Meet está siendo capaz de satisfacerlas. 


\section{METODOLOGíA}

Para dar alcance a dichos objetivos, se adoptó un enfoque metodológico mixto (Creswell y Creswell, 2018), dada la pertinencia que este presenta para analizar el uso de la videoconferencia síncrona en el proceso de enseñanza y aprendizaje universitario (Gegenfurtner et al., 2020).

\section{Contexto y participantes}

Se contó con la participación voluntaria de 52 estudiantes matriculados en una de las asignaturas básicas del $1^{\text {er }}$ curso del Grado de Maestro en Educación Primaria de la Facultad de Educación de la Universidad de Alicante (UA). En cuanto a las variables sociodemográficas, el $71.2 \%$ era menor de 20 años y el $78.8 \%$ de la muestra eran mujeres, lo que resulta coherente con la tradicional feminización que se suele encontrar en este tipo de titulaciones.

\section{Instrumento}

Para la recogida de los datos se utilizó una adaptación del cuestionario de Cabero, Barroso y Llorente (2016). Este es un instrumento elaborado para conocer el grado de adopción de una tecnología a partir del modelo TAM (Technology acceptance model), propuesto inicialmente por Davis (1989). Desde este enfoque, se considera que la aceptación de cualquier herramienta tecnológica se encuentra mediatizada por las creencias que la persona tiene respecto a las consecuencias derivadas de su utilización. Para el estudio de la validez de contenido, la primera adaptación del cuestionario fue sometida a juicio de expertos. En este proceso participaron 3 profesionales con formación en metodología de investigación educativa. En base a su valoración, la primera versión fue rediseñada y pilotada con 10 sujetos de similares características a los participantes potenciales. Tras realizar algunas correcciones gramaticales, se configuró el instrumento definitivo, el cual se organizó en dos bloques temáticos. El primero de ellos tenía como objetivo identificar las necesidades comunicativas que el alumnado universitario experimenta en la modalidad de docencia online impuesta por el COVID-19. El segundo, en cambio, se centraba en analizar el grado de aceptación que los estudiantes muestran hacia Google Meet como herramienta para la enseñanza virtual en situaciones excepcionales.

El bloque inicial quedó integrado por 8 preguntas tipo Likert que se ordenaron, a su vez, en dos dimensiones: (1) tipo de comunicación utilizada por el profesorado durante la suspensión de la actividad docente presencial (4 ítems) y (2) la modalidad de comunicación que, ante estas circunstancias, ellos prefieren (4 ítems). Además de ello, se plantearon 5 preguntas abiertas para conocer las dificultades que habían hallado para comunicarse con el profesorado y con sus compañeros, así 
como los instrumentos empleados para ello. En concreto, estas fueron: (1) ¿Qué recursos tecnológicos ha utilizado tu profesorado para comunicarse? (2) ¿Con qué dificultades te has encontrado para comunicarte con tu profesorado? (3) ¿Qué tipo de comunicación y recursos has utilizado para comunicarte con tus compañeros de clase en general? (4) ¿Con qué dificultades te has encontrado para comunicarte con tus compañeros de clase? (5) ¿Consideras que los recursos utilizados han sido suficientes y adecuados para comunicarte eficazmente con tu profesorado y con tus compañeros de clase, por qué?

La segunda parte, dirigida a conocer el nivel de adopción de Google Meet, se organizó en 6 dimensiones: (1) perfil de uso de Google Meet (2 ítems), (2) facilidad de uso percibida (3 ítems), (3) disfrute percibido (4 ítems), (4) utilidad percibida (6 ítems), (5) actitud de uso (3 ítems) e (6) intención de uso (4 ítems). Se incluyeron, además, 2 preguntas abiertas para reconocer las propuestas que plantean para ajustar la respuesta de la UA a sus necesidades comunicativas, durante el periodo de no presencialidad, y para optimizar el uso de Google Meet en dicho contexto. Específicamente, estas fueron: (1) En base a tu experiencia, ¿qué sugerencias propondrías para mejorar la utilización de Google Meet en la enseñanza universitaria no presencial? (2) ¿Qué otras herramientas y estrategias propondrías para que la universidad desarrollara esta modalidad de enseñanza online de manera más eficaz? Todas las preguntas tipo Likert del cuestionario presentaban una escala de respuesta de 7 puntos, cuyas opciones de respuesta oscilaban desde 1 ("Completamente en desacuerdo") hasta 7 ("Completamente de acuerdo").

La versión definitiva del cuestionario se creó en formato digital mediante Google Forms y se administró de manera telemática. Además de las circunstancias actuales de no presencialidad, el uso de esta herramienta vino motivado por las posibilidades que presenta para la administración de encuestas online en las instituciones de Educación Superior (Sandhya et al., 2020).

\section{Procedimiento}

Como docente de los participantes, una de las investigadoras fue la persona encargada de facilitar al alumnado el enlace al cuestionario durante el horario habitual de la clase virtual. Tras informar a los estudiantes de los objetivos del estudio, así como del carácter confidencial y anónimo de la investigación, esta les proporcionó el link a la encuesta mediante el chat de Google Meet. Para resolver las posibles dudas generadas y para garantizar la correcta interpretación de cada una de las cuestiones planteadas, la investigadora estuvo presente en todo momento en el aula virtual. El tiempo de cumplimentación osciló entre los 30-40 minutos. 


\section{Tratamiento y análisis de la información}

El análisis de los datos se realizó en función de la naturaleza de los mismos. Inicialmente, se llevó a cabo un estudio descriptivo con el programa de análisis estadístico SPSS v. 25. Con posterioridad, y contando con el apoyo del software AQUAD 7 (Huber y Gürtler, 2013), se ejecutó un análisis de contenido convencional y un análisis sumativo (Hsieh y Shannon, 2005). Con el primero de estos, la información fue clasificada y organizada en unidades significativas que, a su vez, formaban parte de categorías con un significado más amplio. Para llevar a cabo la codificación de los datos, se siguieron los pasos de transcripción, agrupación, categorización y abstracción (Elo y Kyngäs, 2008). En base a este proceso, se definió un marco de categorías y códigos con el que se llevó a cabo el análisis de la información, previa validación del instrumento por parte de las profesionales que con anterioridad habían revisado el cuestionario. En última instancia, el análisis sumativo permitió, mediante el recuento de las frecuencias, distinguir las cuestiones que enfatizaron las voces de los participantes.

\section{RESULTADOS}

La presentación de los resultados se hace atendiendo a su naturaleza. Por ello, en primer término se exponen aquellos de carácter cuantitativo y, con posterioridad, los de tipo cualitativo.

\section{Análisis cuantitativo}

Para una mejor comprensión de los datos cuantitativos, su exposición se organiza según las distintas dimensiones que conforman el cuestionario empleado.

\section{Comunicación empleada por el profesorado en la modalidad de enseñanza online}

Tal y como se muestra en la tabla 1, como consecuencia de la suspensión de la actividad docente presencial por el COVID-19 y el desarrollo de una modalidad de enseñanza online, el profesorado parece haber optado por el uso de la comunicación audiovisual, frente a la de carácter textual que ha gozado de un menor nivel de empleo, tanto en su modalidad asíncrona (ítem 4) como, sobre todo, síncrona (ítem 2). Ahora bien, dentro de la comunicación audiovisual ha sido la síncrona la que se ha utilizado más profusamente por parte de los docentes (ítem 1). 
Tabla 1. Tipo de comunicación utilizada por el profesorado en la modalidad de enseñanza online COVID-19

\begin{tabular}{|c|c|c|c|c|c|c|c|c|c|}
\hline Item & $1(\%)$ & $2(\%)$ & $3(\%)$ & $4(\%)$ & $5(\%)$ & $6(\%)$ & $7(\%)$ & $M$ & $D E$ \\
\hline $\begin{array}{l}\text { 1. Comunicación } \\
\text { audiovisual síncrona }\end{array}$ & o & 3.8 & 1.9 & 9.6 & 19.2 & 28.8 & 36.5 & $5 \cdot 77$ & 1.30 \\
\hline $\begin{array}{l}\text { 2. Comunicación textual } \\
\text { síncrona }\end{array}$ & 5.8 & 3.8 & 15.4 & 9.6 & 25 & $17 \cdot 3$ & 23.1 & 4.88 & 1.76 \\
\hline $\begin{array}{l}\text { 3. Comunicación } \\
\text { audiovisual asíncrona }\end{array}$ & o & 9.6 & 5.8 & 15.4 & 25 & $17 \cdot 3$ & 26.9 & 5.15 & 1.58 \\
\hline $\begin{array}{l}\text { 4. Comunicación textual } \\
\text { asíncrona }\end{array}$ & 1.9 & $17 \cdot 3$ & 7.7 & 7.7 & $17 \cdot 3$ & 21.2 & 26.9 & 4.92 & 1.89 \\
\hline
\end{tabular}

\section{Comunicación demandada por el alumnado en la modalidad de enseñanza online}

Este mismo patrón se aprecia en el caso del alumnado, quien también parece mostrar una clara preferencia por la comunicación audiovisual y, dentro de esta, por la de tipo síncrono (ítem 1) (tabla 2). Con respecto a la comunicación textual, vuelve a ser la asíncrona la que tiene menos partidarios entre los estudiantes (ítem 4).

Tabla 2. Tipo de comunicación preferida por el alumnado en la modalidad de enseñanza online COVID-19

\begin{tabular}{|c|c|c|c|c|c|c|c|c|c|}
\hline Ítem & $1(\%)$ & $2(\%)$ & $3(\%)$ & $4(\%)$ & $5(\%)$ & $6(\%)$ & $7(\%)$ & $M$ & $D E$ \\
\hline $\begin{array}{l}\text { 1. Comunicación } \\
\text { audiovisual síncrona }\end{array}$ & o & 3.8 & 3.8 & $7 \cdot 7$ & 7.7 & 25 & 51.9 & 6.02 & 1.37 \\
\hline $\begin{array}{l}\text { 2. Comunicación textual } \\
\text { síncrona }\end{array}$ & 13.5 & 3.8 & 13.5 & 13.5 & 21.2 & 21.2 & 13.5 & 4.42 & 1.91 \\
\hline $\begin{array}{l}\text { 3. Comunicación } \\
\text { audiovisual asíncrona }\end{array}$ & 3.8 & 3.8 & 3.8 & 13.5 & 19.2 & 26.9 & 28.8 & $5 \cdot 37$ & 1.60 \\
\hline $\begin{array}{l}\text { 4. Comunicación textual } \\
\text { asíncrona }\end{array}$ & 19.2 & 9.6 & 9.6 & 15.4 & 23.1 & 19.2 & 3.8 & 3.87 & 1.90 \\
\hline
\end{tabular}

\section{Recursos tecnológicos utilizados por el docente en la comunicación}

De acuerdo con el alumnado, el profesorado se ha valido para la comunicación, sobre todo, de herramientas de videoconferencia síncrona, entre las que destaca especialmente Google Meet (28.30\%) (figura 1). Junto a ello, también ha sido notable el uso de UACloud (18.87\%), el campus virtual de la UA que permite la gestión telemática del proceso de enseñanza y aprendizaje, así como la interacción entre el profesorado y el alumnado mediante las aplicaciones de tutoría y debate. 
Otros recursos que también han sido utilizados por los docentes, pero en menor medida, han sido Zoom (11.95\%), Google Classroom y Moodle, estos dos últimos con idéntico porcentaje de uso (9.43\%). Una tasa más reducida de empleo presenta YouTube (8.80\%) y Adobe Connect (6.30\%).

Figura 1. Recursos tecnológicos utilizados por el profesorado en la comunicación

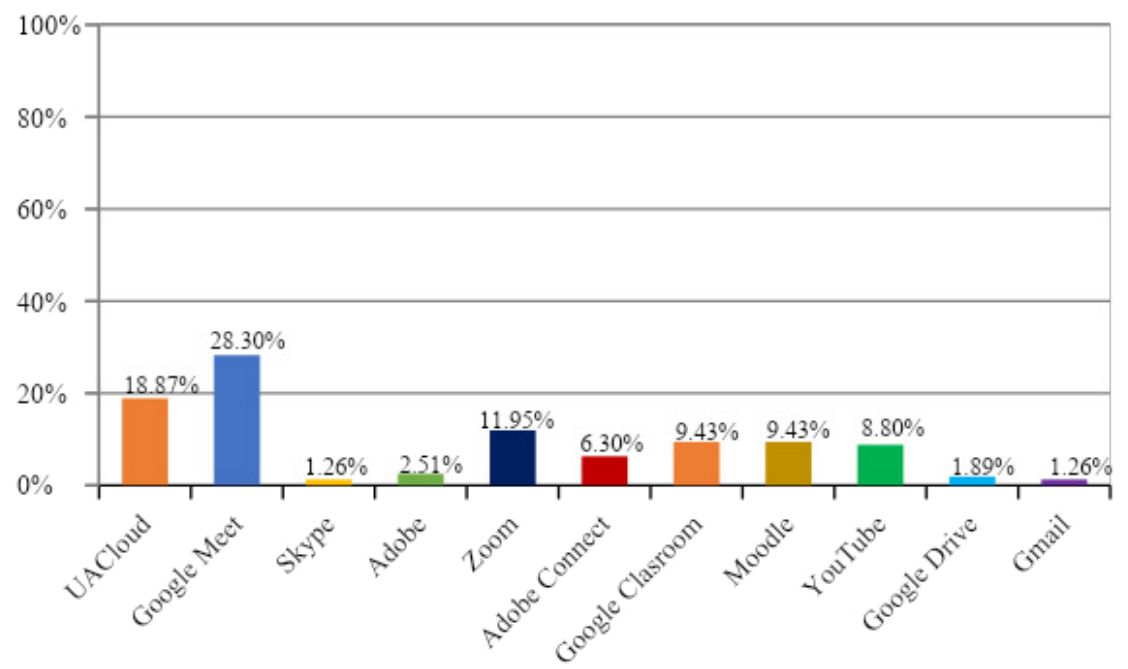

Recursos tecnológicos utilizados por el alumnado en la comunicación con sus compañeros

Frente a esta tendencia docente, para su comunicación con los compañeros de clase el alumnado ha hecho uso, sobre todo, de los servicios de mensajería instantánea y video llamada como Whatsapp (41.76\%) o Skype, aunque este último en mucha menor medida (23.08\%) (figura 2). Del mismo modo, se han servido de Google Drive para la realización de actividades colaborativas (12.09\%) y de Zoom (7.69\%), Instragram (5.49\%) y Google Meet (4.39\%), aunque este último de manera muy escasa y puntual. 
Figura 2. Recursos tecnológicos utilizados por el alumnado para comunicarse con compañeros

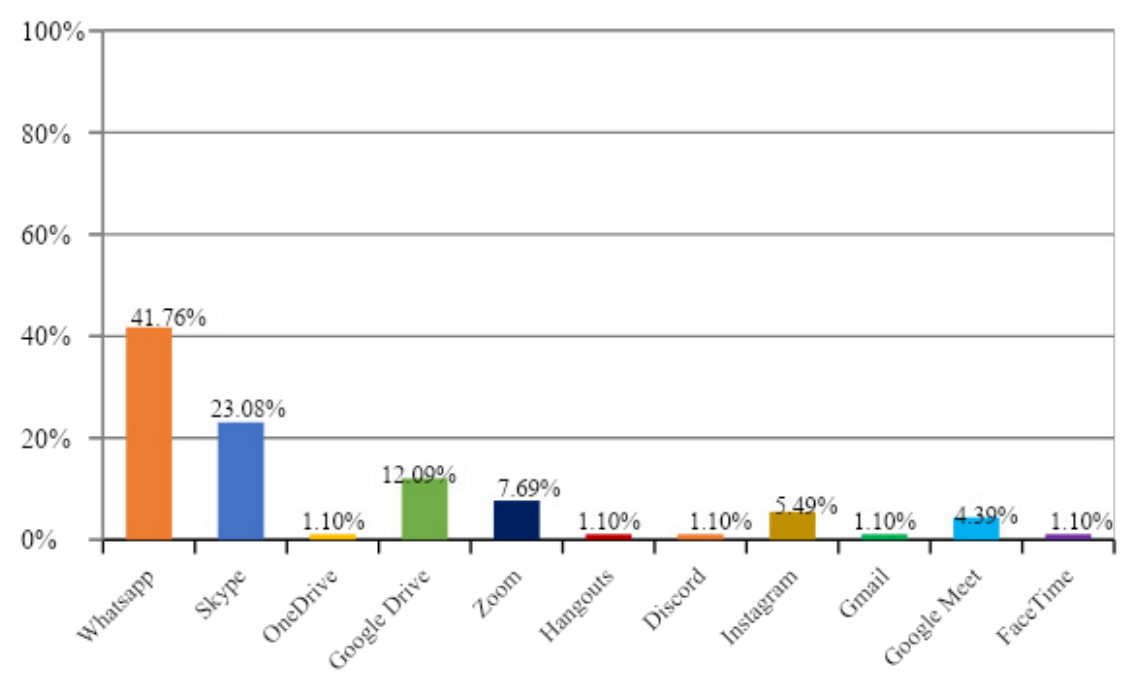

\section{Perfil de uso de Google Meet}

Con anterioridad a la situación excepcional provocada por el COVID-19, prácticamente la totalidad del alumnado reconoce no haber utilizado Google Meet (figura 3).

Figura 3. Uso de Google Meet previo al COVID-19

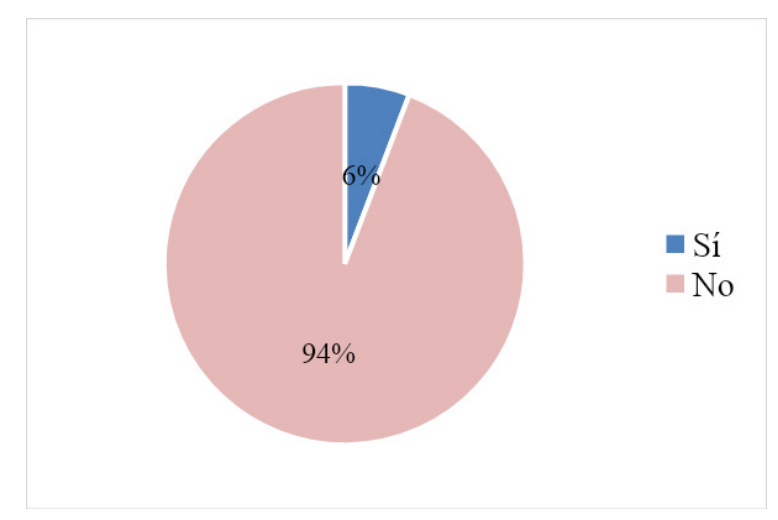


Aunque como consecuencia de la suspensión de las clases presenciales, manifiestan haberla utilizado en la mayoría de las asignaturas que están cursando (figura 4).

Figura 4. Uso de Google Meet durante la docencia online

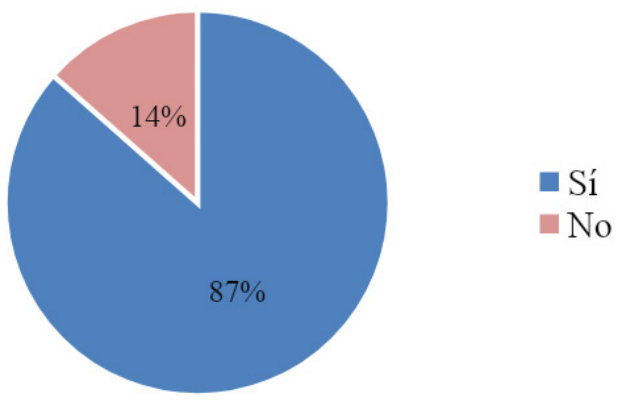

Facilidad de uso percibida en Google Meet

En cuanto a la facilidad de uso, los participantes afirman, de manera categórica, que han encontrado numerosos problemas a la hora de utilizar Google Meet durante este periodo de docencia virtual (ítem 2) (tabla 3). De hecho, señalan que no les ha resultado una aplicación sencilla (ítem 1), pese a reconocer, de forma contundente, que la aplicación tiene una interfaz intuitiva y amigable (ítem 3).

Tabla 3. Facilidad de uso percibida en Google Meet para enseñanza online COVID-19

\begin{tabular}{|c|c|c|c|c|c|c|c|c|c|}
\hline Ítem & $1(\%)$ & 2(\%) & $3(\%)$ & $4(\%)$ & $5(\%)$ & $6(\%)$ & $7(\%)$ & $M$ & $D E$ \\
\hline $\begin{array}{l}\text { 1. Durante este periodo } \\
\text { de actividad no } \\
\text { presencial, Google } \\
\text { Meet me ha resultado } \\
\text { sencillo de utilizar. }\end{array}$ & 5.8 & 94.2 & 0 & 0 & 0 & 0 & 0 & 1.94 & .235 \\
\hline $\begin{array}{l}\text { 2. No he tenido } \\
\text { problemas para } \\
\text { aprender a utilizar } \\
\text { Google Meet a lo largo } \\
\text { de este tiempo de } \\
\text { docencia virtual. }\end{array}$ & 86.5 & 13.5 & 0 & 0 & 0 & 0 & 0 & 1.13 & .345 \\
\hline
\end{tabular}




\begin{tabular}{|c|c|c|c|c|c|c|c|c|c|}
\hline Ítem & $1(\%)$ & $2(\%)$ & $3(\%)$ & $4(\%)$ & $5(\%)$ & $6(\%)$ & $7(\%)$ & $M$ & $D E$ \\
\hline $\begin{array}{l}\text { 3. La interfaz intuitiva, } \\
\text { comprensible y } \\
\text { amigable de Google } \\
\text { Meet me ha facilitado } \\
\text { su empleo durante esta } \\
\text { etapa de aprendizaje } \\
\text { online. }\end{array}$ & 1.9 & 1.9 & 3.8 & 3.8 & 9.6 & 13.5 & 65.4 & 6.19 & 1.42 \\
\hline
\end{tabular}

\section{Disfrute percibido con Google Meet}

Tal y como se recoge en la tabla 4, Google Meet les parece una herramienta divertida y entretenida (ítem 1) e, incluso, interesante y motivadora, teniendo en cuenta las circunstancias de excepcionalidad en las que se hallan, como consecuencia de la suspensión de la enseñanza presencial (ítem 2). Pero con todo, lo cierto es que no parecen mostrar un posicionamiento claramente positivo respecto al grado de disfrute generado (ítem 3) y tampoco acaban de reconocer que su empleo les haya gustado (ítem 4).

Tabla 4. Disfrute percibido con Google Meet para enseñanza online COVID-19

\begin{tabular}{|c|c|c|c|c|c|c|c|c|c|}
\hline Ítem & $1(\%)$ & $2(\%)$ & $3(\%)$ & $4(\%)$ & $5(\%)$ & $6(\%)$ & $7(\%)$ & $M$ & $D E$ \\
\hline $\begin{array}{l}\text { 1. Utilizar Google Meet } \\
\text { como recurso para } \\
\text { la enseñanza virtual } \\
\text { ha sido entretenido y } \\
\text { divertido. }\end{array}$ & 5.8 & 0 & 1.9 & 0 & 13.5 & 21.2 & 57.7 & 6.10 & 1.53 \\
\hline $\begin{array}{l}\text { 2. El uso de Google } \\
\text { Meet me ha resultado } \\
\text { interesante y } \\
\text { motivador, teniendo en } \\
\text { cuenta la suspensión } \\
\text { de la actividad docente } \\
\text { presencial. }\end{array}$ & 3.8 & 1.9 & 1.9 & 11.5 & 11.5 & 28.8 & 40.4 & $5 \cdot 73$ & 1.54 \\
\hline $\begin{array}{l}\text { 3. Dadas las } \\
\text { circunstancias } \\
\text { excepcionales, me ha } \\
\text { gustado utilizar Google } \\
\text { Meet. }\end{array}$ & $7 \cdot 7$ & 11.5 & 3.8 & 17.3 & 32.7 & 13.5 & 13.5 & 4.50 & 1.75 \\
\hline $\begin{array}{l}\text { 4. Me he sentido bien } \\
\text { utilizando Google } \\
\text { Meet. }\end{array}$ & 5.8 & $7 \cdot 7$ & $7 \cdot 7$ & 15.4 & 23.1 & 23.1 & 17.3 & 4.81 & 1.73 \\
\hline
\end{tabular}




\section{Utilidad percibida en Google Meet}

Respecto a la utilidad de la herramienta para la enseñanza virtual en condiciones de excepcionalidad, claramente se advierte que esta les ha resultado un recurso provechoso para optimizar su aprendizaje (ítem 3) (tabla 5). En efecto, los estudiantes manifiestan que Google Meet es una aplicación útil para aprender de manera virtual (ítem 1) y que, gracias a ella, van a mejorar sus resultados en las asignaturas (ítem 4). De hecho, su utilización parece haberles permitido comprender algunos conceptos (ítem 2). En cambio, parecen mostrar un menor grado de acuerdo respecto a las posibilidades que Google Meet les ha ofrecido para estar en contacto con el resto de sus compañeros (ítem 5) y, especialmente, con su profesorado (ítem 6).

Tabla 5. Utilidad percibida en Google Meet para enseñanza online COVID-19

\begin{tabular}{|c|c|c|c|c|c|c|c|c|c|}
\hline Ítem & $1(\%)$ & $2(\%)$ & $3(\%)$ & $4(\%)$ & $5(\%)$ & $6(\%)$ & $7(\%)$ & $M$ & $D E$ \\
\hline $\begin{array}{l}\text { 1. Creo que Google Meet } \\
\text { es útil para aprender } \\
\text { de manera online. }\end{array}$ & 7.7 & 1.9 & 7.7 & 7.7 & 28.8 & 13.5 & 32.7 & 5.19 & 1.80 \\
\hline $\begin{array}{l}\text { 2. Teniendo en cuenta } \\
\text { la suspensión de la } \\
\text { actividad docente } \\
\text { presencial, la } \\
\text { utilización de Google } \\
\text { Meet me ha ayudado } \\
\text { a comprender ciertos } \\
\text { conceptos. }\end{array}$ & 7.7 & 3.8 & 7.7 & 19.2 & 11.5 & 23.1 & 26.9 & 5.00 & 1.85 \\
\hline $\begin{array}{l}\text { 3. El uso de Google Meet } \\
\text { me ha ayudado a } \\
\text { mejorar mi aprendizaje } \\
\text { en esta etapa de } \\
\text { enseñanza virtual. }\end{array}$ & 3.8 & 1.9 & 3.8 & 13.5 & 25 & 26.9 & 25 & $5 \cdot 35$ & 1.50 \\
\hline $\begin{array}{l}\text { 4. Ante la situación } \\
\text { de excepcionalidad } \\
\text { provocada por el } \\
\text { COVID-19, creo que } \\
\text { Google Meet me va a } \\
\text { ayudar a mejorar mi } \\
\text { rendimiento en las } \\
\text { asignaturas. }\end{array}$ & o & $7 \cdot 7$ & $7 \cdot 7$ & $17 \cdot 3$ & 19.2 & 25 & 23.1 & 5.15 & 1.53 \\
\hline
\end{tabular}




\begin{tabular}{|c|c|c|c|c|c|c|c|c|c|}
\hline Ítem & $1(\%)$ & $2(\%)$ & $3(\%)$ & $4(\%)$ & $5(\%)$ & $6(\%)$ & $7(\%)$ & $M$ & $D E$ \\
\hline $\begin{array}{l}\text { 5. Durante este periodo } \\
\text { de no presencialidad, } \\
\text { Google Meet me ha } \\
\text { permitido estar en } \\
\text { contacto con mis } \\
\text { compañeros. }\end{array}$ & 5.8 & 5.8 & 7.7 & $17 \cdot 3$ & 25 & 30.8 & 7.7 & 4.73 & 1.58 \\
\hline $\begin{array}{l}\text { 6. Durante este periodo } \\
\text { de no presencialidad, } \\
\text { Google Meet me ha } \\
\text { ayudado a tener un } \\
\text { contacto más directo } \\
\text { y cercano con mi } \\
\text { profesorado. }\end{array}$ & 9.6 & 7.7 & 13.5 & 25 & 23.1 & $17 \cdot 3$ & 3.8 & 4.12 & 1.61 \\
\hline
\end{tabular}

\section{Actitud de uso hacia Google Meet}

En lo concerniente a la actitud de uso, los participantes señalan que, pese a la excepcionalidad de la situación, el aprendizaje con Google Meet les ha resultado aburrido (ítem 2) y escaso de interés (ítem 1) (tabla 6). De hecho, no acaban de recomendar su empleo como herramienta de aprendizaje online, ni siquiera para circunstancias extraordinarias como las actuales (ítem 3).

Tabla 6. Actitud de uso hacia Google Meet para enseñanza online COVID-19

\begin{tabular}{|c|c|c|c|c|c|c|c|c|c|}
\hline Ítem & $1(\%)$ & $2(\%)$ & $3(\%)$ & $4(\%)$ & $5(\%)$ & $6(\%)$ & $7(\%)$ & $M$ & $D E$ \\
\hline $\begin{array}{l}\text { 1. El uso de Google Meet ha } \\
\text { hecho que el aprendizaje } \\
\text { durante este periodo } \\
\text { excepcional haya sido } \\
\text { más interesante. }\end{array}$ & 17.3 & 11.5 & 13.5 & 21.2 & 13.5 & 11.5 & 11.5 & 3.83 & 1.95 \\
\hline $\begin{array}{l}\text { 2. El empleo de Google } \\
\text { Meet a lo largo de esta } \\
\text { etapa ha provocado que } \\
\text { el aprendizaje haya sido } \\
\text { aburrido. }\end{array}$ & 3.8 & 5.8 & 5.8 & 9.6 & 11.5 & 26.9 & 36.5 & 5.46 & 1.74 \\
\hline $\begin{array}{l}\text { 3. Creo que utilizar Google } \\
\text { Meet como herramienta } \\
\text { de aprendizaje online en } \\
\text { situaciones especiales, } \\
\text { como la provocada por el } \\
\text { COVID-19, es una buena } \\
\text { idea. }\end{array}$ & 3.8 & 11.5 & 9.6 & 30.8 & 17.3 & 21.2 & 5.8 & 4.33 & 1.54 \\
\hline
\end{tabular}




\section{Intención de uso de Google Meet}

Pese a todo ello, manifiestan su deseo de seguir empleando Google Meet cuando la situación se normalice y se retome la enseñanza presencial (tabla 7). Su intención, en ese caso, es utilizarlo para estar en contacto con sus compañeros fuera de clase (ítem 2) y, en menor medida, para interactuar con el profesorado (ítem 3). Ahora bien, lo cierto es que no contemplan su uso en su futuro desempeño profesional (ítem 4) y, menos aún, en su vida académica ulterior (ítem 1).

Tabla 7. Intención de uso de Google Meet para enseñanza online COVID-19

\begin{tabular}{|c|c|c|c|c|c|c|c|c|c|}
\hline Ítem & $1(\%)$ & $2(\%)$ & $3(\%)$ & $4(\%)$ & $5(\%)$ & $6(\%)$ & $7(\%)$ & $M$ & $D E$ \\
\hline $\begin{array}{l}\text { 1. Una vez se retome la } \\
\text { enseñanza presencial, } \\
\text { me gustaría seguir } \\
\text { utilizando Google Meet } \\
\text { en mi vida académica. }\end{array}$ & 5.8 & 13.5 & 26.9 & 30.8 & 9.6 & 7.7 & 5.8 & 3.71 & 1.48 \\
\hline $\begin{array}{l}\text { 2. Una vez se retome la } \\
\text { enseñanza presencial, } \\
\text { me gustaría continuar } \\
\text { utilizando Google Meet } \\
\text { para estar en contacto } \\
\text { con mis compañeros } \\
\text { fuera de clase. }\end{array}$ & 1.9 & 1.9 & 3.8 & 7.7 & 25 & 26.9 & 32.7 & 5.63 & 1.38 \\
\hline $\begin{array}{l}\text { 3. Una vez se retome la } \\
\text { enseñanza presencial, } \\
\text { me gustaría seguir } \\
\text { empleando Google } \\
\text { Meet para estar en } \\
\text { contacto con mi } \\
\text { profesorado fuera de } \\
\text { clase. }\end{array}$ & 9.6 & 9.6 & 7.7 & 26.9 & 23.1 & 11.5 & 11.5 & 4.25 & 1.74 \\
\hline $\begin{array}{l}\text { 4e gustaría utilizar } \\
\text { Google Meet en } \\
\text { mi desempeño } \\
\text { profesional futuro si } \\
\text { tuviera oportunidad, } \\
\text { aunque no se } \\
\text { produzcan situaciones } \\
\text { excepcionales. }\end{array}$ & 17.3 & 5.8 & 17.3 & 28.8 & 11.5 & 7.7 & 11.5 & 3.81 & 1.85 \\
\hline
\end{tabular}




\section{Análisis cualitativo}

A partir del análisis de los discursos de los participantes, emergieron 3 temáticas relacionadas con el paso de la enseñanza presencial a la virtual, como consecuencia del COVID-19: (1) Dificultades que han encontrado en la comunicación durante este periodo, (2) Grado de adecuación de los recursos tecnológicos utilizados por el profesorado y (3) Propuestas de mejora. La exposición de resultados cualitativos obedece al grado de presencia de los códigos y subcódigos en las voces de los estudiantes. Esta se recoge en una serie de tablas donde:

- La frecuencia absoluta (FA) de cada código o subcódigo se refiere al número de veces que los alumnos aluden a una unidad significativa en concreto.

- El porcentaje de frecuencia absoluta (\%FA) de cada código o subcódigo es el resultado del cálculo de FAx100/totalFA.

De manera paralela, se ha de indicar que, para ilustrar y clarificar el significado de cada uno de los códigos y subcódigos empleados en el análisis, la presentación de los resultados se hace acompañada de un conjunto de narrativas.

\section{Dificultades en la comunicación}

Esta primera temática se estructura, a su vez, en dos grandes categorías, referidas a los obstáculos que el alumnado ha encontrado durante el periodo de enseñanza no presencial para comunicarse con el profesorado y con sus compañeros. En cuanto a la primera (tabla 8), los participantes señalan, de manera reiterada, que la principal dificultad que han tenido para contactar con su profesorado ha venido provocada, sobre todo, por una gestión inadecuada de la respuesta educativa ante la nueva situación [Otros profesores han aparecido a las dos semanas de la cuarentena y han intentado que diéramos clases en las vacaciones, Est_04]. Asimismo, hay quienes subrayan el constante envío de mensajes y la demanda excesiva de actividades prácticas y trabajos, lo que les ha acabado generando un elevado nivel de estrés y ansiedad [Hay veces que se cuelga un anuncio comunicando al alumnado que debe de hacer una actividad de hoy a las 8:0o a hoy a las 14:0o. Si por lo que sea esa mañana no has entrado a los anuncios o a lo mejor has tenido clases y hasta las 13:0o no entras a ver los anuncios, no te enteras y has perdido la oportunidad de hacer esa actividad, ya sea voluntaria o no, Est_42]. Los envíos a destiempo de los enlaces a las aulas virtuales también parecen haber obstaculizado, en la práctica, la comunicación y el adecuado desarrollo del proceso de enseñanza y aprendizaje [Pasar el enlace de la videoconferencia a horas tardías, Est_23]. 
Tabla 8. Frecuencias absolutas de los códigos y subcódigos pertenecientes a la Categoría 1.1.

\begin{tabular}{|c|c|c|c|}
\hline Categoria & Códigos & FA & $\%$ FA \\
\hline \multirow{6}{*}{$\begin{array}{l}\text { 1.1. Dificultades en la } \\
\text { comunicación con el profesorado }\end{array}$} & 1.1.1. Técnicas & & \\
\hline & $\begin{array}{l}\text { 1.1.1.1. Mala conexión } \\
\text { 1.1.1.2. Audio }\end{array}$ & $\begin{array}{c}19 \\
6\end{array}$ & $\begin{array}{c}24.66 \\
7.8\end{array}$ \\
\hline & 1.1.2. Atención educativa & & \\
\hline & 1.1.2.1. Resolución dudas & 16 & 20.78 \\
\hline & 1.1.2.2. Gestión aprendizaje & 20 & 25.98 \\
\hline & 1.1.3. Ninguna & 16 & 20.78 \\
\hline Totales & & 77 & 100 \\
\hline
\end{tabular}

Junto a estos problemas, y con una frecuencia acumulada prácticamente similar, se hallan las dificultades causadas por una conexión deficitaria [La comunicación por Google Meet es buena, pero en mi caso en algunas ocasiones falla por mi conexión a internet, Est_36]. Estas limitaciones técnicas han provocado, a su vez, que algunos estudiantes no hayan podido conectarse o seguir las clases virtuales [En mi caso, a algunas clases no he podido asistir por el fallo de internet en mi casa y en otras se me ha cortado la conexión a la mitad, Est_10]. Otro impedimento que mencionan, de forma recurrente, es el relacionado con la resolución de las dudas que les han podido surgir, tanto de manera síncrona como asíncrona [La calidad de la enseñanza online es más problemática que la presencial, ya que en numerosas ocasiones la gente pregunta dudas y a los profesores no les llega el aviso de ningún mensaje y siguen con la explicación, Est_6; Algún docente tarda en contestar mucho a las tutorías y después responde brevemente, Est_38]. Pese a ello, y como consecuencia de la variedad de respuestas entre el profesorado, hay voces que niegan haber experimentado algún tipo de contrariedad [La verdad es que mi profesora se comunica muy bien con nosotros y resuelve nuestras dudas inmediatamente, Est_22]. En estrecha relación con la débil conexión a Internet, aunque con una ocurrencia notoriamente reducida en sus discursos, señalan los problemas de audio [Algunas veces, debido a una mala conexión, se cortaba el audio, Est_05].

Tabla 9. Frecuencias absolutas de los códigos y subcódigos pertenecientes a la Categoría 1.2.

\begin{tabular}{|l|l|c|c|}
\hline \multicolumn{1}{|c|}{ Categoría } & Códigos & FA & \%FA \\
\hline \multirow{4}{*}{$\begin{array}{l}\text { 1.2. Dificultades en la } \\
\text { comunicación con los compañeros }\end{array}$} & 1.2.1. Técnicas & & \\
\cline { 2 - 4 } & $\begin{array}{l}\text { 1.2.1.1. Mala conexión } \\
\text { 1.2.1.2. Audio }\end{array}$ & 14 & 21.21 \\
\cline { 2 - 4 } & 1.2 .2 Brecha digital & 3 & 4.54 \\
\cline { 2 - 4 } & 1.2 .3 . Coordinación & 24 & 13.65 \\
\cline { 2 - 4 } & 1.2 .4 . Ninguna & 66 & 10.36 \\
\hline Totales & & & 24.24 \\
\hline
\end{tabular}


Respecto a las dificultades en la comunicación con los compañeros de clase (tabla 9), los participantes enfatizan, recurrentemente, las barreras que han tenido para coordinar y gestionar el trabajo en equipo [No todos podemos conectarnos al mismo tiempo para realizar prácticas, Est_o9]. La imposibilidad de trabajar de manera presencial parece haber provocado, además, cierto grado de confusión y desconcierto en la realización de las actividades grupales [No es lo mismo hablar y quedar todos en la universidad o en alguna casa que por video llamada puesto que si hablamos todos es imposible entenderse y muchas veces es necesario que unos hablemos con unos y otros con otros y es un lío, Est_07]. Frente a quienes reconocen haber experimentado este tipo de dificultad, también hay quienes afirman no haber encontrado ningún obstáculo [No mucha, ya que seguimos en contacto como de normal: Whatsapp, Instagram, Est_28]. Pero lo cierto es que también con los compañeros, los problemas de carácter técnico parecen haber entorpecido la comunicación, especialmente la mala conexión a Internet y los problemas de audio [Con mucha dificultad, dado que no en todo momento hemos tenido conexión wifi y eso ha complicado mucho las cosas, además no siempre se escucha bien, Est_04].En última instancia, y con el menor porcentaje de frecuencia absoluta, se han destacar los problemas en la comunicación que algunos estudiantes manifiestan haber tenido por no disponer, ellos o sus compañeros, de los recursos tecnológicos necesarios [En mi caso, con mis grupos de trabajo hemos encontrado la dificultad de que solo podemos conectarnos por Whatsapp, ya que una compañera no tiene internet en casa, Est_42]. Además, en algunas ocasiones el alumnado se ha visto obligado a compartir los dispositivos con el resto de miembros de su familia, lo que ha acabado condicionando las posibilidades de trabajo en grupo [Falta de recursos por parte de algunos compañeros, comparten ordenador con sus padres y dependen del horario de estos, Est_32].

\section{Grado de adecuación de los recursos tecnológicos empleados por el profesorado}

La segunda temática, organizada en dos categorías, versa sobre el nivel de pertinencia de las herramientas utilizadas por los docentes para llevar a cabo la enseñanza no presencial. La primera categoría se encarga de agrupar todos aquellos discursos que denotan un cierto grado de conformidad con la respuesta dada por el profesorado (tabla 10).

Tabla 10. Frecuencias absolutas de los códigos pertenecientes a la Categoría 2.1.

\begin{tabular}{|l|l|c|c|}
\hline \multicolumn{1}{|c|}{ Categoría } & \multicolumn{1}{|c|}{ Códigos } & FA & \%FA \\
\hline \multirow{2}{*}{ 2.1. Adecuadas y suficientes } & 2.1.1. Pertinencia herramientas & 25 & 56.82 \\
\cline { 2 - 4 } & 2.1.2. Implicación profesorado & 19 & 43.18 \\
\hline Totales & & 44 & 100 \\
\hline
\end{tabular}


En este contexto, los alumnos consideran que esta adecuación viene motivada porque los recursos empleados son los más indicados, sobre todo teniendo en cuenta la excepcionalidad de la situación [Considerando que esta situación ha sido bastante inesperada y excepcional, considero que los recursos que están siendo utilizados están siendo suficientes y adecuados, ya que permiten, aunque sea de forma telemática, poder seguir dando clase casi de la misma forma que yendo a la universidad, Est_o3]. Frente a ello, y con una tasa de frecuencia absoluta más reducida, también hay quienes consideran que el ajuste de la respuesta está provocado, más bien, por la dedicación y la implicación del profesorado, quien se hace cargo de la situación y se esfuerza por atender y satisfacer sus necesidades [Sí, puesto que la profesora ha mantenido desde el primer momento de esta situación tan complicada el contacto con los alumnos desde todo tipo de aplicaciones y siempre que tienes una duda te la responde a los pocos días, Est_41]. Ahora bien, lo cierto es que quienes se sitúan en este posicionamiento dejan entrever en sus relatos una cierta actitud de resignación [No hay otra forma de hacerlo, hay que trabajar a base de conferencias y explicar mucho las cosas porque no sabemos qué va a ocurrir, Est_14].

Tabla 11. Frecuencias absolutas de los códigos pertenecientes a la Categoría 2.2.

\begin{tabular}{|l|l|c|c|}
\hline \multicolumn{1}{|c|}{ Categoría } & \multicolumn{1}{|c|}{ Códigos } & FA & \%FA \\
\hline \multirow{2}{*}{ 2.2. Inapropiadas e insuficientes } & 2.2.1. Fallos funcionamiento & 15 & 46.88 \\
\cline { 2 - 4 } & $\begin{array}{l}\text { 2.2.2. Falta de implicación } \\
\text { docente }\end{array}$ & 17 & 53.12 \\
\hline Totales & & 32 & 100 \\
\hline
\end{tabular}

La segunda categoría, en cambio, se ocupa de aglutinar todas aquellas voces que no consideran válidos los recursos empleados por el profesorado para adaptar la enseñanza a la modalidad virtual (tabla 11). En este caso, sus narraciones destacan, sobre todo, la escasa implicación docente como causa principal de su inadecuación [No, no me parecen suficientes. Creo que no están haciendo las cosas bien y eso se nota, no es lo mismo estar en una clase presencial que en una online y si a eso le sumas, que quieren que te estudies tu solito el temario de una clase para otra y que en la clase online solo quieras preguntar lo que ha quedado claro, creo que no es lo adecuado, Est_02]. A pesar de ello, y con una similar tasa de ocurrencia, también hay testimonios que consideran que esta inadecuación radica en los problemas de funcionamiento que presentan algunas de las herramientas utilizadas [Dependiendo de la asignatura. Por ejemplo, en esa y en la otra han utilizado aplicaciones que a mucha gente nos da fallo para entrar y para permanecer dentro, Est_23]. 


\section{Propuestas de mejora}

La tercera de las temáticas, relativa a las sugerencias de mejora que el alumnado plantea para optimizar la modalidad de enseñanza online, se estructura en dos grandes categorías. Respecto a las ideas para optimizar el uso de Google Meet, las voces de los participantes parecen rechazar la formulación de posibles sugerencias (tabla 12). Este posicionamiento se debe, fundamentalmente, a que lo consideran un recurso válido para el aprendizaje virtual [Creo que es una herramienta bastante completay por ahora no me hadadoningún problema,Est_25]. Dehecho, reconocen que gracias a esta herramienta han podido continuar su proceso de formación [En general, la utilización de Google Meet ayuda a poder continuar con las clases, por lo que no propondría ninguna sugerencia, Est_49]. Ahora bien, también hay quienes prefieren no hacer ningún tipo de sugerencia por considerar que este no se ajusta a las necesidades que están experimentando [Es que creo que Google Meet no sirve, ya que es difícil mantener una conversación a través de una videoconferencia con tantas personas, y es por eso que la mayor parte de los alumnos nos sentimos inútiles a la hora de las clases, e inconscientemente, dejamos de prestar atención a la explicación, Est_24].

Tabla 12. Frecuencias absolutas de los códigos y subcódigos pertenecientes a la Categoría 3.1

\begin{tabular}{|c|c|c|c|}
\hline Categoria & Códigos & FA & $\%$ FA \\
\hline \multirow{10}{*}{$\begin{array}{l}\text { 3.1. Para el uso de Google Meet en } \\
\text { la enseñanza universitaria online }\end{array}$} & 3.1.1. Sugerencias técnicas & & \\
\hline & 3.1.1.1. Mejora sonido & 8 & 14.28 \\
\hline & $\begin{array}{l}\text { 3.1.1.2. Integración de } \\
\text { pizarra }\end{array}$ & 2 & 3.58 \\
\hline & $\begin{array}{l}\text { 3.1.1.3. Uso de } \\
\text { notificaciones }\end{array}$ & 6 & 10.71 \\
\hline & 3.1.2. Sugerencias al profesorado & & \\
\hline & 3.1.2.1. Grabar sesión & 5 & 8.93 \\
\hline & $\begin{array}{l}\text { 3.1.2.2. Fomentar } \\
\text { interacción }\end{array}$ & 10 & 17.86 \\
\hline & 3.1.3. Ninguna & & \\
\hline & 3.1.3.1. Adecuada & 19 & 33.93 \\
\hline & 3.1.3.2. Inadecuada & 6 & 10.71 \\
\hline Totales & & 56 & 100 \\
\hline
\end{tabular}

Frente a esta tendencia, también hay quienes consideran que es responsabilidad del profesorado mejorar el uso de Google Meet en la enseñanza virtual, planteándole a este sus propuestas. Entre otras, le recomiendan dinamizar el aprendizaje y fomentar la interacción entre los alumnos [Propongo organizar debates por videoconferencia 
para participar y que sea más ameno y para comprender mejor los contenidos, Est_08], así como grabar las clases y ponerlas, posteriormente, a disposición del alumnado [En primer lugar, que cada uno de los profesores que haga uso de esta aplicación grabase la clase. Esto supondría una gran ayuda para los estudiantes, ya que podrían acudir tantas veces como quisieran para tomar apuntes o solventar cualquier duda posible, Est_07].

En última instancia, subrayan las mejoras de carácter técnico, entre las que parecen enfatizar la necesidad de optimizar la calidad del sonido [Quitar el ruido de múltiples voces a la vez, Est_09;], la incorporación de alguna aplicación a modo de pizarra [Pizarras donde pueda escribir el profesor como hace en clase, Est_31] y el uso de algún servicio de notificaciones para los mensajes del chat [Mejoraría algunos aspectos de la aplicación, como la manera de ver los comentarios, ya que si compartes pantalla no puedes leer los comentarios, Est_21].

Tabla 13. Frecuencias absolutas de los códigos y subcódigos pertenecientes a la Categoría 3.2

\begin{tabular}{|c|c|c|c|}
\hline Categoría & Códigos & FA & $\%$ FA \\
\hline \multirow{8}{*}{$\begin{array}{l}\text { 3.2. Para la mejora de la } \\
\text { enseñanza online }\end{array}$} & 3.2.1. Uso de herramientas & & \\
\hline & $\begin{array}{l}\text { 3.2.1.1. Síncronas } \\
\text { 3.2.1.2. Asíncronas }\end{array}$ & $\begin{array}{c}28 \\
6\end{array}$ & $\begin{array}{c}30.77 \\
6.59\end{array}$ \\
\hline & 3.2.2. Estrategias & & \\
\hline & 3.2.2.1. Planificación & 13 & 14.28 \\
\hline & $\begin{array}{l}\text { 3.2.2.2.Respuesta brecha } \\
\text { digital }\end{array}$ & 2 & 2.20 \\
\hline & $\begin{array}{l}\text { 3.2.2.3. Equilibrar carga } \\
\text { trabajo }\end{array}$ & 36 & 39.56 \\
\hline & 3.2.2.4. Formación docente & 2 & 2.20 \\
\hline & 3.2.3. Ninguna & 4 & 4.40 \\
\hline Totales & & 91 & 100 \\
\hline
\end{tabular}

La segunda y última categoría se ocupa de compilar todas aquellas voces que sugieren propuestas para mejorar la modalidad de aprendizaje virtual que ha impuesto el COVID-19 (tabla 13). En este contexto, los discursos de los participantes parecen enfatizar la conveniencia de utilizar, sobre todo, herramientas que favorezcan una comunicación síncrona [Adobe Connect, que es la herramienta que he usado en esa otra asignatura, también me resulta bastante buena para realizar clases online, Est_03]. Pese a ello, también hay algunos testimonios que recomiendan, aunque con menor insistencia, el uso de recursos de tipo asíncrono [Otra estrategia podía ser YouTube y subir videos explicativos del tema, Est_36].

En cuanto a las estrategias de mejora, los alumnos proponen de manera categórica reducir la carga de trabajo académico, ya que afirman sentirse ostensiblemente 
sobrepasados [Que los profesores no se pasen con los trabajos puesto que estamos hasta arriba de tareas y aunque estemos en casa no podemos estar cada día y a todas horas mirando la plataforma de UACloud, Est_14]. También apuntan la necesidad de mejorar la planificación y coordinación de la respuesta que, desde la institución, se está dando a la situación, especialmente en lo relativo al uso de una única herramienta tecnológica [Cómo bien he mencionado anteriormente, que todos los profesores hagan uso de la misma plataforma web, aplicación, etc., Est_07]. En último término, y con similar porcentaje de frecuencia, exigen la solución de los problemas relacionados con la brecha digital [Estrategias que tengan en cuenta a las personas que no tienen un acceso fácil a las tecnologías requeridas para las clases online, Est_27], así como incidir en el desarrollo de la competencia digital del profesorado [Creo que el profesorado debería estar preparado para dar clases online, y esto es una cuestión que ahora no se puede remediar, creo yo. Entonces para que esta situación sea lo más llevadera posible para todos debemos poner tanto alumnado como profesorado de nuestra parte para que este método funcione, Est_43].

\section{DISCUSIÓN Y CONCLUSIONES}

Reconociendo la importancia que las herramientas tecnológicas tienen para dar respuesta a los problemas que enfrenta el ser humano y para el avance y la mejora de la sociedad, esta investigación se planteó con el propósito de identificar las necesidades de comunicación que el alumnado universitario está teniendo en la modalidad de enseñanza online impuesta por el COVID-19 y, en segunda instancia, reconocer si Google Meet está siendo capaz de satisfacerlas. A la vista de los hallazgos arrojados por el estudio, el proceso de adaptación a la virtualidad no se está produciendo en las mejores condiciones, al menos en lo que respecta al alumnado. De acuerdo con este, aunque el profesorado está haciendo uso de una modalidad síncrona de enseñanza, lo que se corresponde con sus necesidades, la sobrecarga de trabajo y la falta de una adecuada planificación y gestión de la respuesta educativa están dificultando en gran medida su proceso de aprendizaje (Franchi, 2020). A ello se ha de unir, además, el impacto de las dificultades que están encontrando los estudiantes para comunicarse con sus compañeros, algo que intentan paliar con las aplicaciones y herramientas con las que están familiarizados, como Whatsapp (So, 2016).

En lo concerniente al uso de Google Meet, este no parece venir a dar respuesta a sus necesidades, ya que aunque lo consideran un recurso útil y valioso para el aprendizaje virtual, su utilización les genera ciertas dificultades, sobre todo como consecuencia de la falta de conocimientos, los problemas de conexión y el retardo entre la imagen y el sonido (Budiana y Yutanto, 2020); limitaciones que también han sido puestas de manifiesto con el uso de otras aplicaciones similares (Cornelius, 2014; Morrison, 2011). En definitiva, y con el propósito de optimizar la respuesta que desde las instituciones de Educación Superior se está dando a la situación 
excepcional que estamos atravesando, se concluye señalando la conveniencia de atender sus necesidades y, en base a estas, rediseñar el plan de actuación con el que la universidad va a hacer frente a uno de sus mayores desafíos.

\section{REFERENCIAS}

Barbosa, S., y Amariles, M. (2019). Learning styles and the use of ICT in university students within a competencybased training model. Journal of New Approaches in Educational Research, 8(1), 1-6. https://doi.org/10.7821/ naer.2019.1.296

Budiana, K. M., y Yutanto H. (2020). Webinar: A strategy to enhance speaking skill of non-native English speaker. Journal of English Educators Society, 5(1), 31-37. https://doi.org/10.21070/ jees.v5i1.354

Cabero, J., Barroso, J., y Llorente, Ma ${ }^{\mathrm{a}}$ C. (2016). Technology acceptance model \& augmented reality: Study in progress. Revista Lasallista de Investigación, 13(2), 18-26. https://doi.org/10.22507/ rli.v13n2a2

Carrick, F. R., Abdulrahman, M., Hankir, A., Zayaruzny, M., Najem, K., Lungchukiet, P., y Edwards, R. A. (2017). Randomized controlled study of a remote flipped classroom neuro-otology curriculum. Frontiers in Human Neuroscience, 8, 349. https://doi.org/10.3389/ fneur.2017.00349

Cecilio-Fernandes, D., Parisi, M. C. R., Santos, T. M., y Sandars, J. (2020). The COVID-19 pandemic and the challenge of using technology for medical education in low and middle income countries. MedEdPublish. https://doi. org/10.15694/mep.2020.000074.1

Cendon, E. (2018). Lifelong learning at universities: Future perspectives for teaching and learning. Journal of New Approaches in Educational Research, 7(2), 81-87. http://dx.doi.org/10.7821/ naer.2018.7.320
Chen, C. H., Liao, C. H., Chen, Y. C., y Lee, C. F. (2011). The integration of synchronous communication technology into service learning for pre-service teachers' online tutoring of middle school students. Internet and Higher Education, 14, 27-33. https://doi.org/10.1016/j. iheduc.2010.02.003

Chen, J., Dobinson, T., y Kent, S. (2020). Student's perspectives on the impact of Blackboard Collaborate on Open University Australia (OUA) online learning. Journal of Educators Online, 17(1). https://bit.ly/3by9qM3. https:// doi.org/10.1080/02680513.2019.168865 4

Cornelius, S. (2014). Facilitating in a demanding environment: Experiences of teaching in virtual classrooms using web conferencing. British Journal of Educational Technology, 45(2), 260-271. https://doi.org/10.1111/bjet.12016

Creswell, J. W., y Creswell, J. D. (2018). Research design: Qualitative, quantitative, and mixed methods approaches. Sage.

Davis, F. D. (1989). Perceived usefulness, perceived ease of use, and user acceptance of information technology. MIS Quarterly, 13(3), 319-340. https://doi. org/10.2307/249008

Elo, S., y Kyngäs, H. (2008). The qualitative content analysis process. Journal of Advanced Nursing, 62(1), 107115. $\quad$ https://doi.org/10.1111/j.13652648.2007.04569.x

Franchi, T. (2020). The impact of the COVID-19 pandemic on current anatomy education and future careers: A student's perspective. Anatomical Sciences 
Education. https://doi.org/10.1002/ ase.1966

Gegenfurtner, A., Zitt, A., y Ebner, C. (2020). Evaluating webinar-based training: A mixed methods study of trainee reactions toward digital web conferencing. International Journal of Training and Development, 24(1), 5-21. https://doi. org/10.1111/ijtd.12167

Hsieh, H. F., y Shannon, S. E. (2005). Three approaches to qualitative content analysis. Qualitative Health Research, 15(9), 1277-1288. https://doi. org/10.1177/1049732305276687

Huber, G. L., y Gürtler, L. (2013). AQUAD 7. Manual: The analysis of qualitative data. Ingeborg Huber Verlag.

Infante-Moro, A., Infante-Moro, J., y Gallardo-Pérez, J. (2019). The importance of ICTs for students as a competence for their future professional performance: The case of the faculty of business studies and tourism of the University of Huelva. Journal of New Approaches in Educational Research, 8(2), 201-213. http://dx.doi.org/10.7821/ naer.2019.7.434

Kerres, M. (2020). Against all odds: Education in Germany coping with COVID-19. Postdigital Science and Education. $\quad$ https://doi.org/10.1007/ s42438-020-00130-7

Morrison, K. A. (2011). Using web conferencing to teach constructivist, discussion-rich seminars: Can it work? The Quarterly Review on Distance Education, 12(4), 269-274.

Rao, P. (2019). Webinars and their effective use in English language teaching and learning. ELT Vibes: International E-Journal for Research in ELT, 5(1), 7397.

Sandars, J., Correia, R., Dankbaar, M., de Jong, P., Goh, P. S., Hege, I. ... Pusic, M. (2020). Twelve tips for rapidly migrating to online learning during the COVID-19 pandemic. MedEdPublish. https://doi. org $/ 10.15694 / \mathrm{mep} .2020 .000082 .1$

Sandhya, S., Koppad, S. H., Kumar, S. A., Dharani, A., Uma, B. V., y Subramanya, K. N. (2020). Adoption of Google Forms for enhancing collaborative stakeholder engagement in higher education. JEET Journal of Engineering Education Transformations, $33, \quad 283-289$. https://doi.org/10.16920/jeet/2020/ v33io/150161

So, S. (2016). Mobile instant messaging support for teaching and learning in higher education. Internet and Higher Education, 31, 32-42. https://doi. org/10.1016/j.iheduc.2016.06.001

Taha, M. H, Abdalla, M. E., Wadi, M., y Khalafalla, H. (2020). Curriculum delivery in medical education during an emergency: A guide based on the responses to the COVID-19 pandemic. MedEdPublish. https://doi. org/10.15694/mep.2020.000069.1

Trespalacios, J., y Uribe-Florez, L. (2020). Developing online sense of community: Graduate students' experiences and perceptions. Turkish Online Journal of Distance Education, 21(1), 57-72. https:// doi.org/10.17718/tojde.690340

UNESCO. (2020). Handbook on facilitating flexible learning during educational disruption: The Chinese experience in maintaining undisrupted learning in COVID-19 outbreak. https://bit. ly/3fQyeSJ

Villalón, R., Luna, Ma ${ }^{\mathrm{a}}$, y García-Barrera, A. (2019). Valoración y uso de la plataforma Blackboard Collaborate en una universidad a distancia: estudio de caso sobre las prácticas declaradas de docentes del Grado de Psicología. Digital Education Review, 35, 267-288. 


\section{PERFIL ACADÉMICO Y PROFESIONAL DE LAS AUTORAS}

Rosabel Roig-Vila: Catedrática de Universidad de Tecnología Educativa (CU) de la Universidad de Alicante y Doctora en Pedagogía (premio extraordinario). Ha sido Decana de la Facultad de Educación de la UA (2005-2009) y actualmente es Directora del Instituto de Ciencias de la Educación de esta universidad. Es editora de la revista electrónica científica Journal of New Approaches in Educational Research (NAER) (http://www.naerjournal.ua.es) y dirige el grupo de investigación GIDUEDUTIC/IN. https://orcid.org/0000-0002-9731-430X

E-mail: rosabel.roig@ua.es

Mayra Urrea-Solano: Profesora asociada del Departamento de Didáctica General y Didácticas Específicas de la Facultad de Educación de la Universidad de Alicante. Forma parte dela Red de Investigación en Diseñoy Oportunidades de Género en la Educación Superior (https://bit.ly/3bVFIRY) y del grupo de investigación GIDU-EDUTIC/IN (http://bit.ly/2Ovbafw). Sus líneas de investigación se centran en el estudio de la tecnología educativa, la igualdad de género y el liderazgo en la Educación Superior. https://orcid.org/0000-0001-8650-7854

E-mail: mayra.urrea@ua.es

Gladys Merma-Molina: Profesora de la Universidad de Alicante. Doctora por la misma universidad. Directora de la Red de Investigación en Diseño y Oportunidades de Género en la Educación Superior (https://bit.ly/3bVFIRY). Sus investigaciones se centran en la igualdad de género, la convivencia y la educación en valores. Investigadora del Grupo de Investigación GIDU.EDUTIC/IN (http:// bit.ly/2Ovbafw) y asesora de Proyectos Iberoamericanos, en la perspectiva de la igualdad de género y la convivencia en la educación. Para obtener más información: https://bit.ly/3aQgZNP.https://orcid.org/0000-0002-9856-6314

E-mail: gladys.merma@ua.es

Dirección:

Facultad de Educación

Universidad de Alicante

Calle Aeroplano, s/n

San Vicente del Raspeig (España)

Fecha de recepción del artículo: 15/05/2020

Fecha de aceptación del artículo: 11/07/2020

Fecha de aprobación para maquetación: 31/08/2020 